\title{
Performance Study of the Association TCM-UGM/STBC to Reduce Transmission Errors of JPEG Images
}

\author{
Mohamed Benaissa $^{{ }^{*}}$, Abdesselam Bassou ${ }^{2}$, Mohammed Beladgham ${ }^{1}$, Abdelmounaim Moulay Lakhdar ${ }^{1}$ \\ ${ }^{1}$ University of Bechar, Bechar, Algeria \\ ${ }^{2}$ Telecommunication and Digital signal Processing Laboratory, Djillali Liabes University, Sidi Bel Abbes, Algeria \\ E-mail: moh.benaissa@gmail.com \\ Received February 24, 2011; revised March 20, 2011; accepted March 31, 2011
}

\begin{abstract}
The purpose of this work is to associate the channel encoder called 'trellis-coded modulation with Ungerboeck-Gray mapping' (TCM-UGM) to 'space-time block code’ (STBC), in order to study its performance to correct the transmission errors of a JPEG image. The performance of the proposed scheme is evaluated in senses of bit error rate (BER), frame error rate (FER) and peak signal-to-noise ratio (PSNR) of the reconstructed image. Compared to the association TCM/STBC for a throughput of $2 \mathrm{bits} / \mathrm{s} / \mathrm{Hz}$, TCM-UGM/STBC permits to obtain a PSNR gain up to $2 \mathrm{~dB}$.
\end{abstract}

Keywords: Trellis-Coded Modulation, Trellis Coded Modulation with Ungerboeck-Gary Mapping, Logarithm of Maximum a Posteriori, Space-Time Block Code, JPEG

\section{Introduction}

In future wireless communication systems, high data rates need to be reliably transmitted over time-varying band limited channels. The wireless channel mainly suffers from time-varying fading due to multipath propagation and destructive superposition of signal received over different paths. Fortunately, the effects of fading can be substantially mitigated by the use of diversity. Different transmit diversity techniques have been introduced. In [1], Tarokh et al. proposed space-time trellis coding by jointly designing the channel coding, modulation, transmit diversity and the optional receiver diversity. The proposed space-time trellis codes perform extremely well at the cost of high complexity. In addressing the issue of decoding complexity, Alamouti [2] discovered a remarkable scheme for transmissions using two transmit antennas. A simple decoding algorithm was introduced, which can be generalised to an arbitrary number of receive antennas. This scheme is significantly less complex, than space-time trellis coding using two transmit antennas, although there is a loss in performance [3]. Despite the associated performance penalty, Alamouti's scheme is appealing in terms of simplicity and performance. This proposal motivated Tarokh et al. [3,4] to generalise the scheme to an arbitrary number of transmit antennas, leading to the concept of space time block codes. Space- time block codes were designed for achieving the maximum diversity order of $n \times m$ for $n$ transmit and $m$ receive antennas. However, they were not designed for achieving additional coding gain.

Hence, in this contribution, we combine space-time block codes with Trellis Coded Modulation (TCM) [5,6], and TCM-UGM [7] in order to achieve additional coding gains. The simulation results showed that the TCMUGM outperforms the original TCM scheme proposed by Ungerboeck by $2.59 \mathrm{~dB}$ over Rayleigh fading channel [7]. The comparison is done at a Bit Error Rate (BER) of 10 - 5. Visual signals such as compressed still images are very vulnerable to channel noise.

Usually, channel coding is utilized to protect the transmitted visual signals. The Joint Photograph Experts Group (JPEG) standard [8] proposed in 1992 is widely used for still image compression and transmission. JPEG has 4 distinct modes of operation: sequential DCT-based, progressive DCT-based, lossless, and hierarchical [9]. JPEG, a DCT-based image compression algorithm [10], is the current ISO standard for the encoding of still images. The JPEG algorithm follows a block-based compression approach. It divides the input image into $8 \times 8$ pixel blocks, transforms each block using DCT, and then codes the DC and AC coefficients. In this paper, we provide an efficient scheme for transmitting JPEG compressed images using the concatenation of STBC with 
TCM-UGM system (TCM-UGM/STBC). The considered image is compressed using JPEG compression algorithm then coded with TCM-UGM/STBC or TCM/STBC. At the receiver, symbol-by-symbol MAP TCM-UGM or TCM decoding algorithm is applied, and the image is reconstructed by decompression algorithm.

\section{Space-Time Block Codes}

A Space Time Block Code describing the relationship between the original transmitted signal and the signal replicas artificially created at the transmitter for transmission over various diversity channels is defined by an $n_{T} x p$ dimensional transmission matrix. The entries of the matrix are constituted of linear combinations of the $k$-airy input symbols $x_{1}, x_{2}, \cdots, x_{k}$ and their conjugates. The k-airy input symbols $x_{i} i=1 \cdots k$ are used to represent the information-bearing binary bits to be transmitted over the transmit diversity channels. In a signal constellation having $2^{m}$ constellation points, a number $\mathrm{m}$ of binary bits are used to represent a symbol $x_{i}$. Hence, a block of kxm binary bits are entered into the STB encoder at a time and it is, therefore, referred to as a STB code. The number of transmitter antennas is $n_{T}$ and $\mathrm{p}$ represents the number of time slots used to transmit $k$ input symbols. Hence, a general form of the transmission matrix of a STBC is written as

$$
\left(\begin{array}{ccccc}
g_{11} & g_{21} & \cdots & \cdots & g_{p 1} \\
g_{12} & g_{22} & \cdots & \cdots & g_{p 2} \\
\vdots & \vdots & \vdots & \vdots & \vdots \\
g_{1 n} & g_{2 n} & \cdots & \cdots & g_{p n}
\end{array}\right)
$$

where the entries $g_{i j}$ represent linear combinations of the symbols $x_{1}, x_{2}, \cdots, x_{k}$ and their conjugates. More specifically, the entries $g_{i j}$, where $x_{i}, i=1, \cdots, n_{T}$ are transmitted simultaneously from transmit antennas $1, \cdots, n_{T}$ in each time slot $j=1, \cdots, p$.

The transmission matrix in Equation (1) (which defines the STBC) is based on a complex generalized orthogonal design, as defined in [3]. Since there are $k$ symbols transmitted over $p$ time slots, the code rate of the STBC is given by

$$
\text { Rate }=\frac{k}{p}
$$

At the receiving end, one can have an arbitrary number of $n_{R}$ receivers. A simple transmit diversity scheme for two transmit antennas was introduced by Alamouti in [2]. The transmission matrix is

$$
G_{2}=\left(\begin{array}{cc}
x_{1} & x_{2} \\
-\bar{x}_{2} & \bar{x}_{1}
\end{array}\right)
$$

It can be seen in the transmission matrix $\boldsymbol{G}_{2}$ that there are $n_{T}=2$ (number of columns in the matrix $\boldsymbol{G}_{2}$ ) transmitters, $k=2$ possible input symbols, namely, $x_{1}, x_{2}$ and the code spans over $p=2$ (number of rows in the matrix $\boldsymbol{G}_{2}$ ) time slots. Since $k=2$ and $p=2$, the code rate is unity. The associated encoding and transmission process is shown in Table 1. At any given time instant $T$, two signals are transmitted simultaneously from the antennas $T x_{1}$ and $T x_{2}$. For example, in the first time slot $T=1$, signal $x_{1}$ is transmitted from antenna $T x_{1}$ and signal $x_{2}$ is transmitted simultaneously from antenna $T x_{2}$. In the next time slot $T=2$, signals $-\bar{x}_{2}$ and $\bar{x}_{1}$ (the conjugates of symbols $x_{1}$ and $x_{2}$ ) are simultaneously transmitted from antennas $T x_{1}$ and $T x_{2}$, respectively.

Figure 1 shows the base band representation of a simple two-transmitter STBC, namely, that of the $\boldsymbol{G}_{2}$ code seen in Equation (3) using one receiver. We can see from the Figure 1 that there are two transmitters, namely, $T x_{1}$ as well as $T x_{2}$ and they transmit two signals simultaneously. As it can be seen from the Figure 1, the transmitted symbol $x_{1}$ and $x_{2}$ propagates through two different fading channels, namely, $h_{1}$ and $h_{2}$. As mentioned earlier,

Table 1. Encoding and transmission process for the STBC.

\begin{tabular}{ccc}
\hline & Antenna \\
\hline Time slot, $T$ & $T x_{1}$ & $T x_{2}$ \\
1 & $x_{1}$ & $x_{2}$ \\
2 & $-\bar{x}_{2}$ & $\bar{x}_{1}$ \\
\hline
\end{tabular}

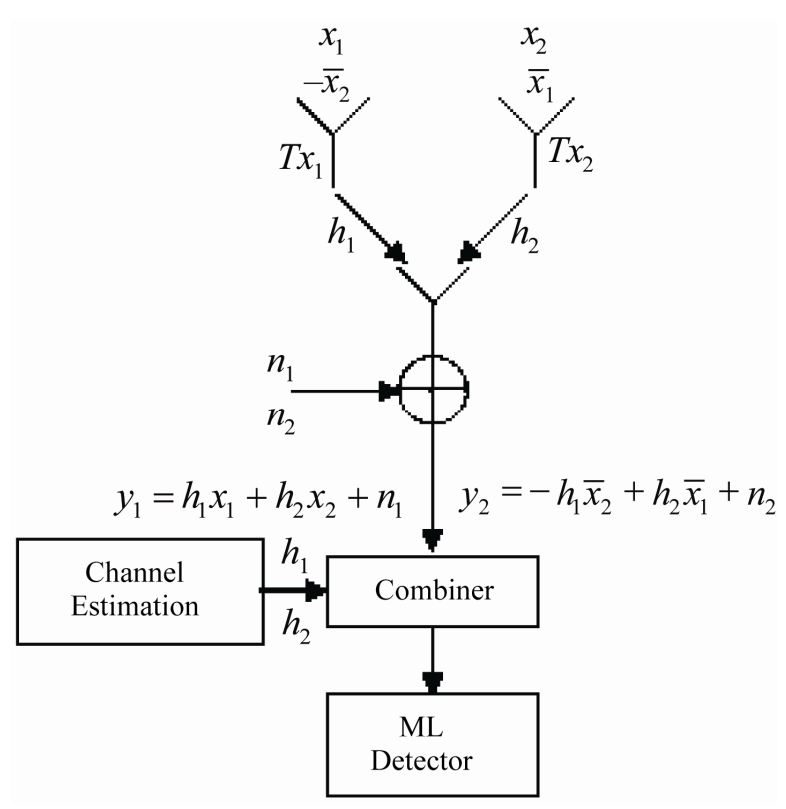

Figure 1. Base band representation of the simple two transmitters STBC $G_{2}$ of (3) using one receiver. 
the complex fading envelope is assumed to be constant across the corresponding two consecutive time slots.

$$
\begin{aligned}
& h_{1}=h_{1}(T=1)=h_{1}(T=2) \\
& h_{2}=h_{2}(T=1)=h_{2}(T=2)
\end{aligned}
$$

At the receiver, independent noise samples, $n_{1}$ and $n_{2}$ are added in each time slot; hence the signals received over non dispersive or narrow-band channels can be expressed with the aid of Equation (3) as

$$
\begin{aligned}
& y_{1}=h_{1} x_{1}+h_{2} x_{2}+n_{1} \\
& y_{2}=-h_{1} \bar{x}_{2}+h_{2} \bar{x}_{1}+n_{2}
\end{aligned}
$$

where $y_{1}$ is the first received signal and $y_{2}$ is the second. Note that the received signal $y_{1}$ consists of the transmitted signals $x_{1}$ and $x_{2}$, while $y_{2}$ consists of their conjugates. In order to determine the transmitted symbols, we have to extract the signals $x_{1}$ and $x_{2}$ from the received signals $y_{1}$ and $y_{2}$. Therefore, both signals $y_{1}$ and $y_{2}$ are passed to the combiner, as shown in Figure 1. In the combineraided by the channel estimator, which provides perfect estimation of the diversity channels in this example simple signal processing is performed in order to separate the signals $x_{1}$ and $x_{2}$. Both signals $x_{1}$ and $x_{2}$ are then passed to the maximum likelihood detector of Figure 1, based on the Euclidean distances between the combined signal $x$ and all possible transmitted symbols. The simplified decision rule is based on choosing $x_{i}$ if and only if

$$
\operatorname{dist}\left(\hat{x}, x_{i}\right) \leq \operatorname{dist}\left(\hat{x}, x_{j}\right) \quad \forall i \neq j
$$

where $\operatorname{dist}(A, B)$ is the Euclidean distance between signals $A$ and $B$ and the index $j$ spans all possible transmitted signals. From Equation (8), we can see that maximum likelihood transmitted symbol is the one having the minimum Euclidean distance from the combined signal $\hat{x}$.

\section{Trellis Coded Modulation with Ungerboeck-Gray Mapping}

The TCM scheme, proposed by Ungerboeck, was designed for throughput of $m$ bits/sec/Hz, where $m$ bits are input to the encoder (among input bits $\tilde{m} \geq 0$ are uncoded) and $m+1$ bits are output and mapped with $2^{m+1}$-airy modulation using set partitioning yielding a coding rate

$$
R=\frac{m}{m+1}
$$

In this case the mapping by set partitioning (called also Ungerboeck mapping) is applied. The Ungerboeck TCM encoder was chosen by maximizing $d_{f}$. In [5], $d_{f}$ is compute by an algorithm that replaces the search in TCM trellis for the path that maximizes $d_{f}$. In [7], the
TCM-UGM scheme considered, for a throughput of $m$ bits/sec/Hz, that the mapper is a $2^{m+1}$-airy that uses a mapping technique combining Ungerboeck mapping and Gray code mapping. In this case, $m-\tilde{m}$ bits are input to the encoder, and systematically output and mapped using Ungerboeck mapping. The Gray mapping is applied on the remaining $\tilde{m}(\tilde{m} \geq 1)$ uncoded bits and the generated parity bit. When no uncoded bits are considered $(\tilde{m}=0)$, this scheme is equivalent to Ungerboeck TCM scheme with $\tilde{m}=0$. In [7], the optimal encoder codegenerator is obtained by searching in the trellis, of two different paths (with a minimum Euclidean distance) that begin at state zero and finish to the same state. The optimal code-generator is obtained by maximizing $d f$. In this work, the TCM-UGM encoder, illustrated in Figure 2, considers $m=2$ input bits of which no uncoded bits are considered $(\tilde{m}=0)$.

\section{Proposal System}

Figure 3 represents the image transmission system for which we evaluate the FER and PSNR after decoding. An input image is compressed by JPEG. The binary symbols resulting from the JPEG compressing constitute the sequence of data to be transmitted. The information data is first encoded by the TCM or TCM-UGM encoder exposed in section 3. The complex constellation symbols, generated by mapper, are interleaved and fed into the space-time block encoder described in section 2 (with $n_{T}$ transmit and $n_{R}$ receive antennas). At each time slot T, the output symbols $x_{i}$ are modulated and transmitted simultaneously each from a different transmit antenna. At the receiver end, the received noisy symbol is decoded using space-time block decoder and deinterleaved giving a soft-decision exploited by TCM or TCM-UGM decoder and is used for the reconstruction image.

\section{Simulation Result}

The performance simulation of the associations TCM/

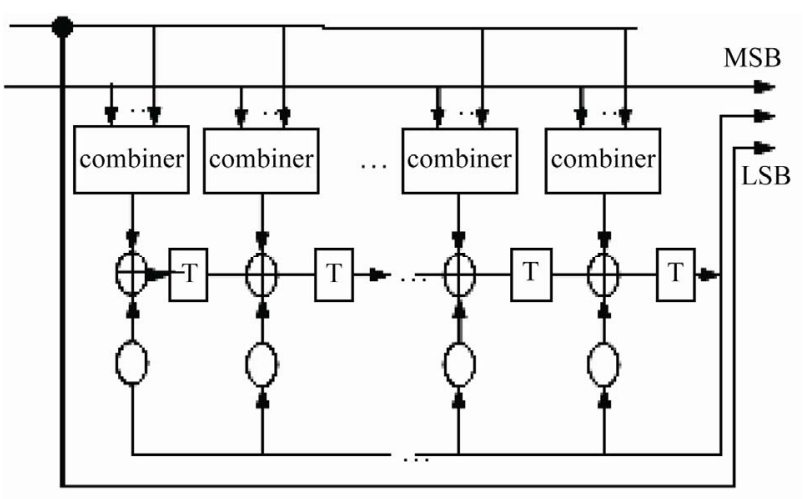

Figure 2. TCM-UGM encoder for 2 bits/s/Hz. 

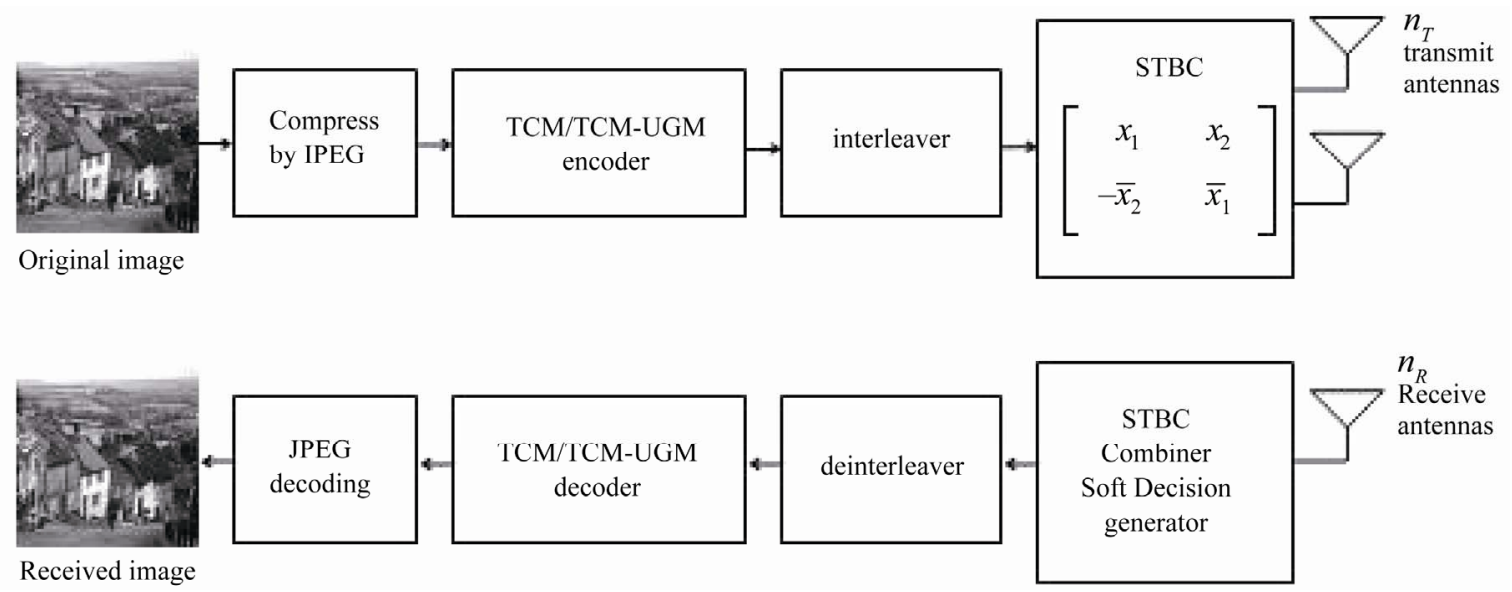

Figure 3. A block diagram of a communication system, $n_{T}=2 ; n_{R}=1$.

STBC and TCM-UGM/STBC using 8PSK Ungerboeck mapper (for TCM) and Ungerboeck-Gray mapper (for TCM-UGM) are investigated for throughput $2 \mathrm{bit} / \mathrm{s} / \mathrm{Hz}$ for JPEG image transmission. Rate $2 / 3$ and 16-state TCM or TCM-UGM encoder is considered. Transmission over MRF channel, using one receiver antenna, is simulated employing STBC with $\boldsymbol{G}_{2}$ as orthogonal code. The optimal encoders' code-generator (in sense of $d_{f}$ ) for the used TCM and TCM-UGM encoders are illustrated in Table 2 (the average power per dimension in the constellation is normalized to $1 / 2$ ).

In simulation, a variety of raw images with high resolution (512 × 512 pixels) are used (boat image in Figure 9, goldhill image in Figure 12 and concord aerial in Figure 15). The images transmitted have different bit per pixel (bpp) (Table 3) to illustrate the effectiveness of the proposed system

The performance of the encoding schemes is evaluated in terms of BER (Bit Error Rate) and FER (Frame Error Rate) versus bit energy to noise ratio $\left(E_{b} / N_{0}\right)$. The FER computation considers a frame length of 1024 .

Figures 4 and 5 illustrate the performance in sense of BER and FER, respectively, of TCM/STBC and TCM-UGM/STBC considering one receiver antenna for the transmission of this JPEG images. It can be observed

Table 2. Code-generator for throughput $2 \mathrm{bits} / \mathrm{s} / \mathrm{Hz}$ STBC encoding and transmission process for the STBC.

\begin{tabular}{ccccccc}
\hline & \multirow{2}{*}{$d_{f}^{2}$} & \multirow{2}{*}{$\begin{array}{c}\text { Memory } \\
\text { order }\end{array}$} & \multicolumn{3}{c}{ Code-generator } \\
\cline { 4 - 6 } TCM & & 4.172 & 4 & 31 & 14 & 30 \\
TCM-UGM & 5.172 & 4 & 23 & 34 & 15 \\
\hline
\end{tabular}

Table 3. Bit Per Pixel rate for different images.

\begin{tabular}{cccc}
\hline Image & Concord-aerial & Boat & Goldhill \\
Bit per pixel & 0.5 & 0.4 & 0.61 \\
\hline
\end{tabular}

that the system TCM-UGM/STBC presents better results than the TCM/STBC from a $E_{b} / N_{0}$ of $10 \mathrm{~dB}$ for BER curves and $7 \mathrm{~dB}$ for FER curves. The TCM-UGM/STBC system outperforms the performance of the association TCM/STBC by $0.4 \mathrm{~dB}$ at BER $=10^{-5}$ and $0.9 \mathrm{~dB}$ at FER $=10^{-3}$.

The Peak Signal-to-Noise Ratio (PSNR) is the most commonly used as a measure of quality of reconstruction in image compression. The PSNR were identified using the following formulae:

$$
\operatorname{MSE}^{2}=\frac{1}{\operatorname{MxN}} \sum_{i=1}^{i=N} \sum_{j=1}^{j=M}(I(i, j)-\hat{I}(i, j))^{2}
$$

Mean Square Error (MSE) which requires two $M x N$ grayscale images $I$ and $\hat{I}$ where one of the images is considered as a compression of the other is defined as: The PSNR is defined as:

$$
\text { PSNR }=10 \log _{10}\left(\frac{(\text { Dynamics of image })^{2}}{\text { MSE }}\right)
$$

Usually an image is encoded on 8 bits. It is represented by 256 gray levels, which vary between 0 and 255, the extent or dynamics of the image is 255 .

Figure 6 to Figure 8 illustrate the performance curves of the PSNR of the reconstructed image vs $E_{b} / N_{0}$. From these figures, it can be shown clearly that the proposed system based on TCM-UGM gives better performance. For an $E_{b} / N_{0}$ of $11 \mathrm{~dB}$ a PSNR improvement of around 2 $\mathrm{dB}$ is obtained for Boat and Goldhill images and around $0.6 \mathrm{~dB}$ to the Concord-aerial image.

Figures 10 and 11 represent, respectively, the reconstructed Boat image after transmission using the TCM-UGM/STBC and TCM/STBC for $E_{b} / N_{0}$ equals 11 $\mathrm{dB}$; we can observe a clear visual improvement made by the proposed system. The same remark can be done for the other images (Figure 13 and Figure 14 for the Goldhill 


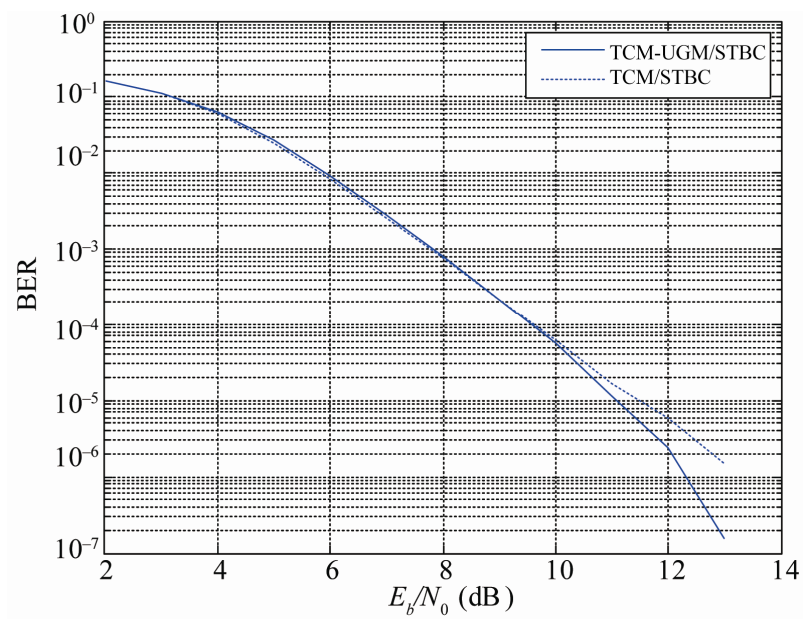

Figure 4. BER performance of TCM/STBC and TCMUGM/STBC schemes over MRF channel $\left(n_{R}=1\right)$.

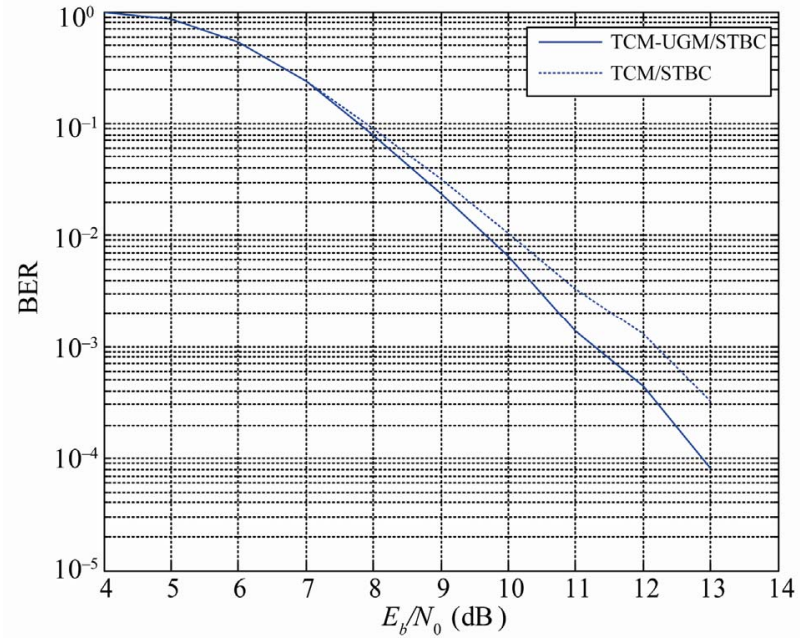

Figure 5. FER performance of TCM/STBC and TCMUGM/STBC schemes over MRF channel $\left(n_{R}=1\right)$.

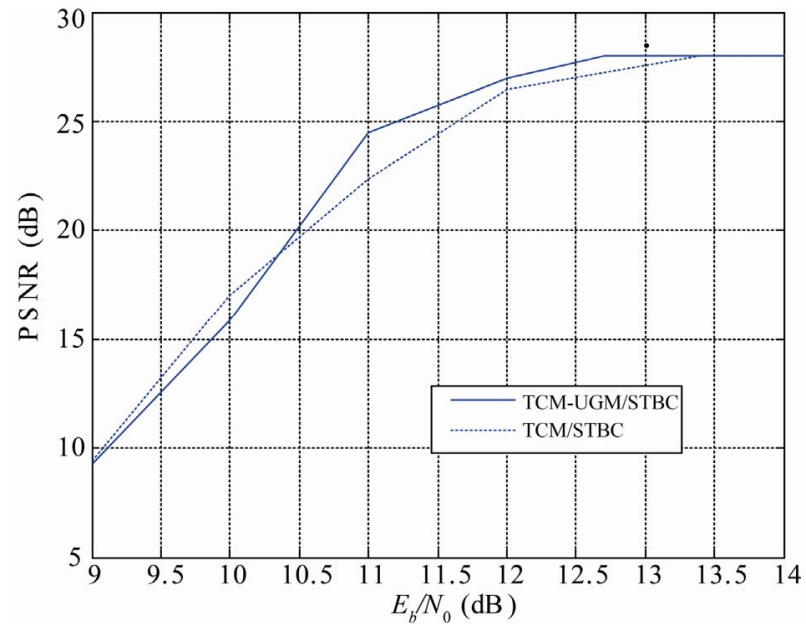

Figure 6. Performance PSNR vs $E_{b} / N_{0}$ for JPEG boat image transmission bpp $=0.4$.

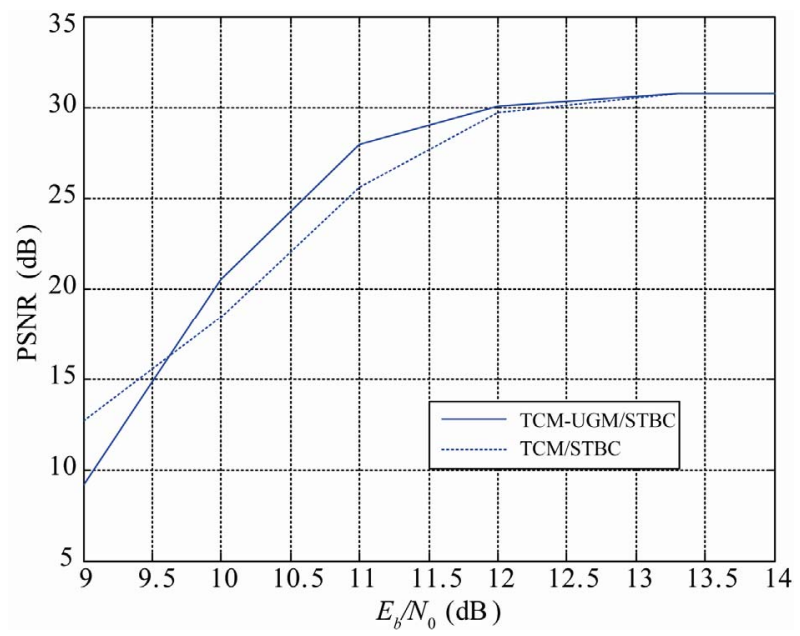

Figure 7. Performance PSNR vs $E_{b} / N_{0}$ for JPEG goldhill image transmission bpp $=0.61$.

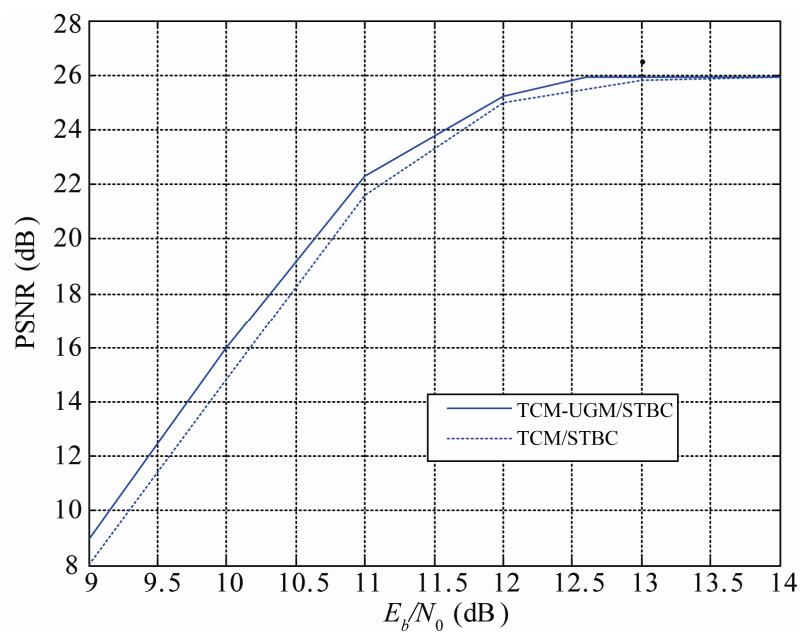

Figure 8. Performance PSNR vs $E_{b} / N_{0}$ for JPEG concordaerial image transmission bpp $=\mathbf{0 . 5}$.

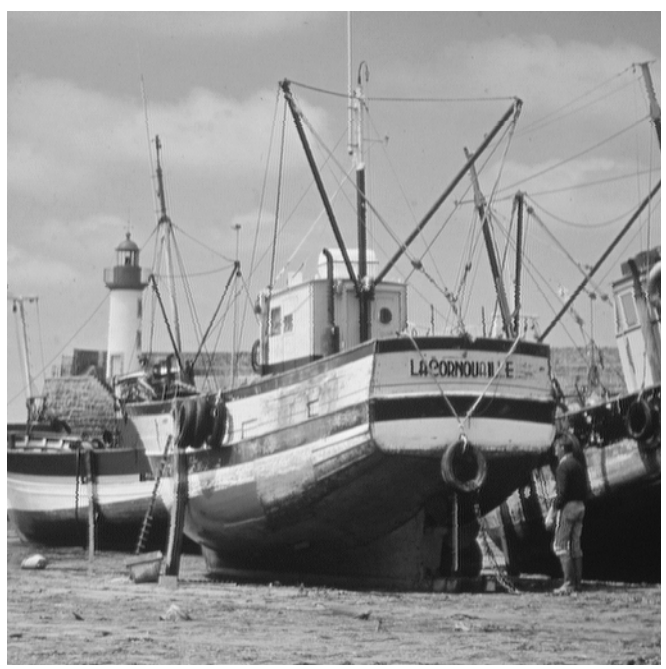

Figure 9. Original Boat image. 


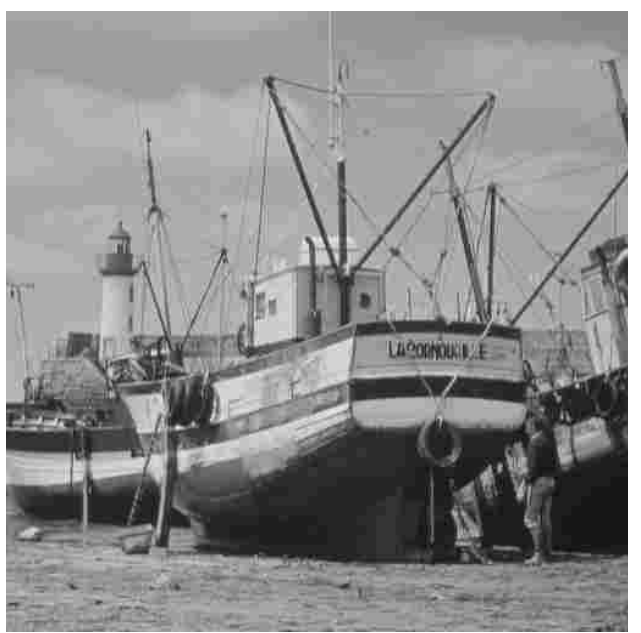

Figure 10. Reconstructed Boat image for TCM-UGM/STBC, bpp $=0.4, E_{b} / N_{0}=11 \mathrm{~dB}$, PSNR $=24.52 \mathrm{~dB}$.

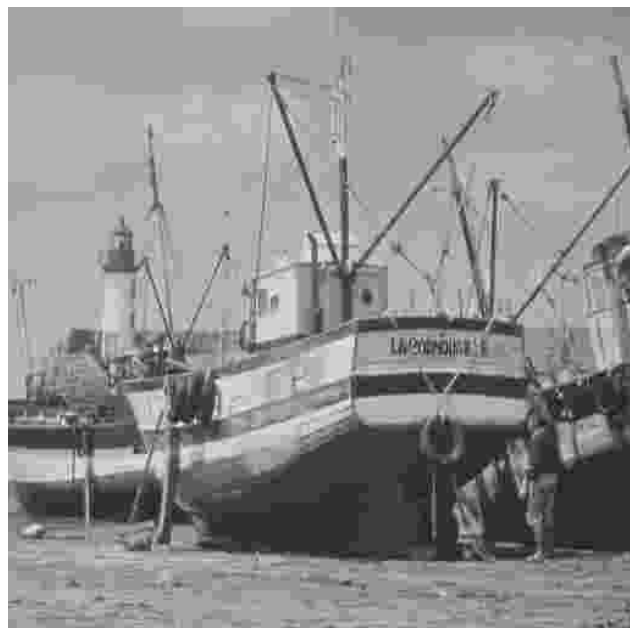

Figure 11. Reconstructed Boat image for TCM/STBC, bpp $=0.4, E_{b} / N_{0}=11 \mathrm{~dB}$, PSNR $=22.28 \mathrm{~dB}$.

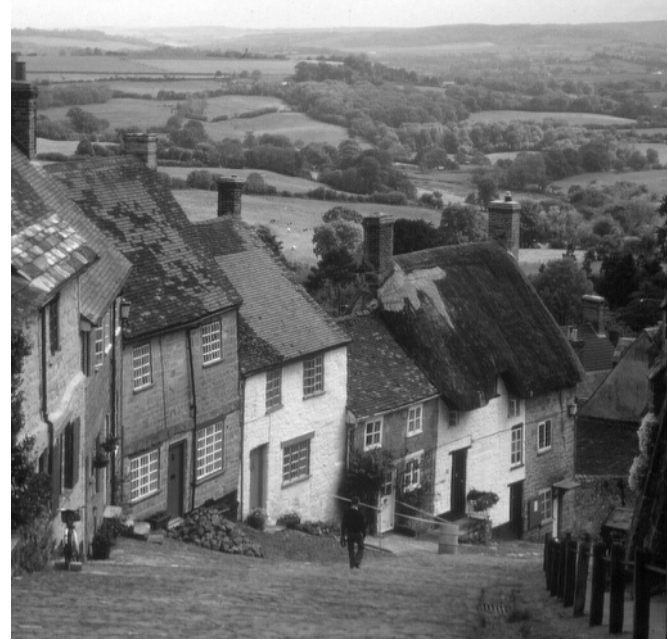

Figure 12. Original goldhill image.

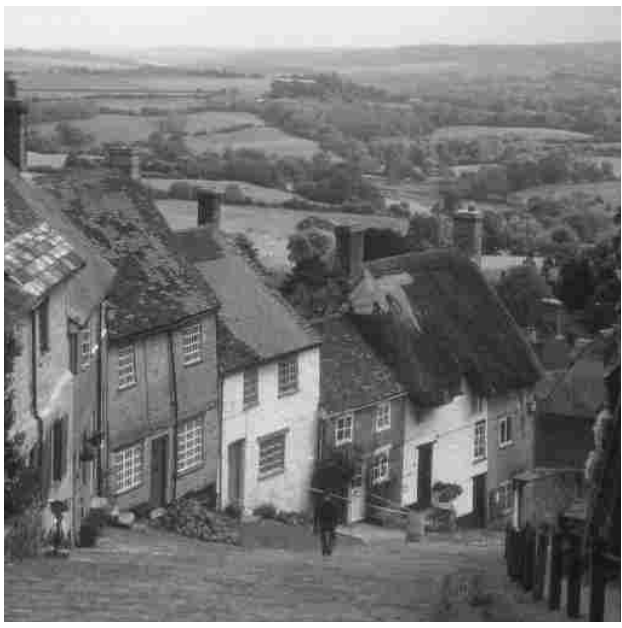

Figure 13. Reconstructed Goldhill image for TCM-UGM/ STBC bpp $=0.61, E_{b} / N_{0}=11 \mathrm{~dB}$, PSNR $=27.92 \mathrm{~dB}$.

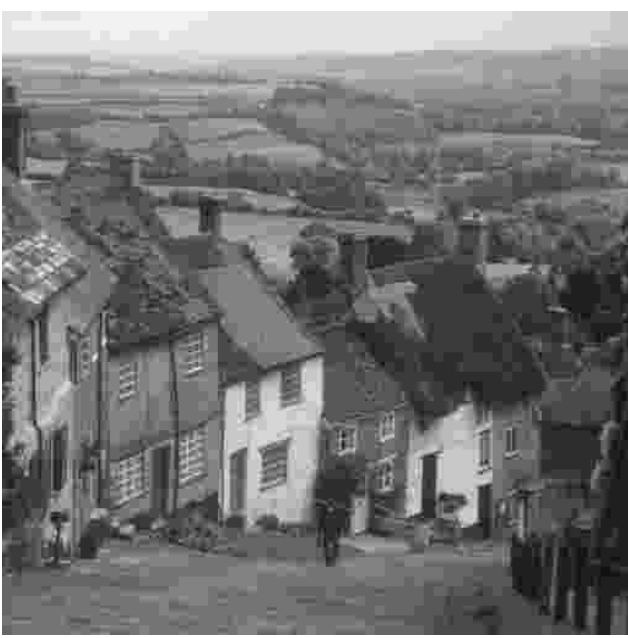

Figure 14. Reconstructed Goldhill image for TCM/STBC bpp $=0.61, E_{b} / N_{0}=11 \mathrm{~dB}$, PSNR $=25.58 \mathrm{~dB}$.

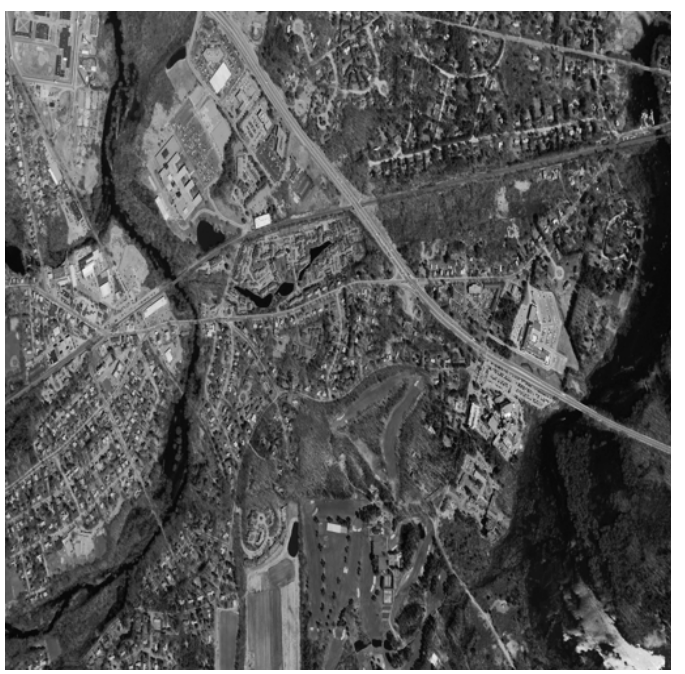

Figure 15. Original concord-aerial image. 


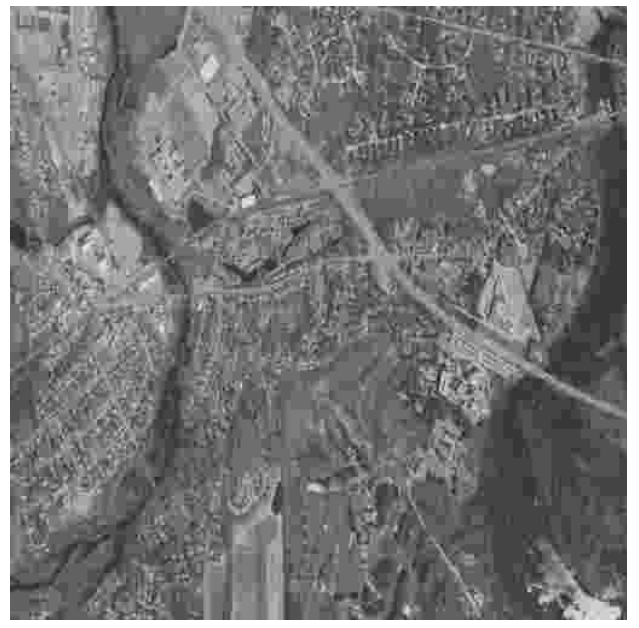

Figure 16. Reconstructed concord-aerial image for TCMUGM/STBC bpp $=0.5, E_{b} / N_{0}=11 \mathrm{~dB}$, PSNR $=22.26 \mathrm{~dB}$.

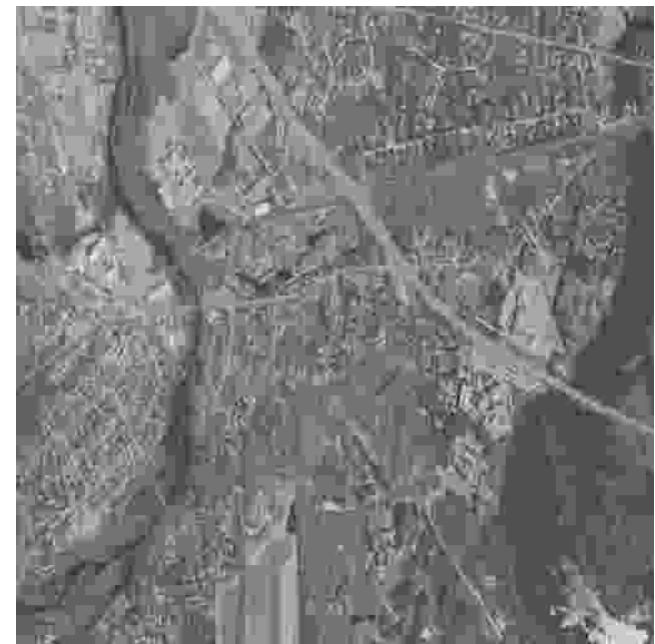

Figure 17. Reconstructed concord-aerial image for TCM/ STBC bpp $=0.5, E_{b} / N_{0}=11 \mathrm{~dB}$, PSNR $=21.58 \mathrm{~dB}$.

image and, Figure 16 and Figure 17 for the Concordaerial image).

\section{Conclusions}

In this work, TCM-UGM/STBC encoding scheme has been used to correct compressed JPEG images transmission errors. The simulation results over MRF channel have shown that the proposed scheme offers better per- formance in sense of FER and PSNR of the reconstructed images, compared to TCM/STBC scheme. For a throughput of $2 \mathrm{bits} / \mathrm{s} / \mathrm{Hz}$ and an $E_{b} / N_{0}$ of $11 \mathrm{~dB}$, TCM$\mathrm{UGM} / \mathrm{STBC}$ permits to obtain a PSNR gain of $2 \mathrm{~dB}$.

\section{References}

[1] V. Tarokh, N. Seshadri and A. R. Calderbank, "SpaceTime Codes for High Data Rate Wireless Communication: Performance Criterion and Code Construction," IEEE Transactions on Information Theory, Vol. 44, No. 2, 1998, pp. 744-765. doi:10.1109/18.661517

[2] S. M. Alamouti, “A Simple Transmit Diversity Technique for Wireless Communications," IEEE Journal on Selected Areas in Communications, Vol. 16, No. 8, 1998, pp. 1451-1458. doi:10.1109/49.730453

[3] V. Tarokh, H. Jafarkhani and A. R. Calderbank, "SpaceTime Block Codes from Orthogonal Designs," IEEE Transactions on Information Theory, Vol. 45, No. 5, 1999, pp. 1456-1467. doi:10.1109/18.771146

[4] V. Tarokh, H. Jafarkhani and A. R. Calderbank, "SpaceTime Block Coding for Wireless Communications: Performance Results," IEEE Journal on Selected Areas in Communications, Vol. 17, No. 3, 1999, pp. 451-460.

[5] G. Ungerboeck, "Channel Coding with Multilevel/Phase Signals," IEEE Transactions on Information Theory, Vol. 28, No. 1, 1982, pp. 55-67. doi:10.1109/TIT.1982.1056454

[6] L. T. Hanzo, H. Liew and B. L. Yeap, "Turbo Coding, Turbo Equalisation and Space Time Coding for Transmission over Wireless Channels,” John Willy IEEE Press, New York, 2002. doi:10.1002/047085474X

[7] A. Bassou and A. Djebbari, "Contribution to the Improvement of the Performance of Trellis-Coded Modulation," WSEAS Transactions on Communications, Vol. 6, No. 2, 2007, pp. 307-311.

[8] "Digital Compression and Coding of Continuous tone Still Images Part 1: Requirements and Guidelines,” International Telecommunication Union, 1991.

[9] "Digital Compression and Coding of Continuous Tone still Images: Requirements and Guidelines,” International Standard Organization, 1994.

[10] G. Lakhani, "DCT Coefficient Prediction for JPEG Image Coding Image Processing," IEEE International Conference on Image Processing, San Antonio, 16 September-19 October 2007, pp. 189-192. doi:10.1109/ICIP.2007.4379986 